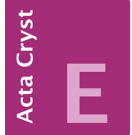
COMMUNICATIONS

ISSN 2056-9890

\section{Crystal structure of $4,4^{\prime}$-[(1,3,5,7-tetra- oxo-1,3,3a,4,4a,5,7,7a,8,8a-decahydro- 4,8-ethenopyrrolo[3,4-f]isoindole-2,6-di- yl)bis(methylene)]bis(pyridin-1-ium) \\ dinitrate}

\section{Zhimin Liu}

School of Chemistry and Chemical Engineering, Shanxi University, Taiyuan 030006, People's Republic of China. *Correspondence e-mail: luckzhmliu@sxu.edu.cn

Received 6 November 2015; accepted 20 November 2015

Edited by H. Stoeckli-Evans, University of Neuchâtel, Switzerland

In the title salt, $\mathrm{C}_{24} \mathrm{H}_{22} \mathrm{~N}_{4} \mathrm{O}_{4}{ }^{2+} \cdot 2 \mathrm{NO}_{3}{ }^{-}$, the cation is U-shaped with the two isoindole dione rings inclined to one another by $60.41(13)^{\circ}$, while the two outer pyridine rings are inclined to one another by $2.77(12)^{\circ}$. The dihedral angles between the pyridine ring and the adjacent isoindole dione ring are $71.82(12)$ and $86.44(13)^{\circ}$. In the crystal, each nitrate anion is linked to a protonated pyridine ring by $\mathrm{N}-\mathrm{H} \cdots \mathrm{O}$ hydrogen bonds. These units are linked by a series of $\mathrm{C}-\mathrm{H} \cdots \mathrm{O}$ hydrogen bonds, forming a three-dimensional structure.

Keywords: crystal structure; salt; isoindole; pyrrolo; pyridinium; nitrate(V) salt; $\mathrm{N}-\mathrm{H} \cdots \mathrm{O}$ hydrogen bonds.

CCDC reference: 1434635

\section{Related literature}

For the crystal structures of compounds with similar ligands, see: Yu et al. (2012); Li et al. (2011, 2012a,b). For the synthetic method used to prepare 2,6-bis(pyridin-4-ylmethyl)3a,4,4a,7a,8,8a-hexahydro-4,8-ethenopyrrolo[3,4-f]isoindole1,3,5,7(2H,6H)-tetraone, see: Liu et al. (2007).<smiles>O=C1C2C3C=CC(C2C(=O)N1Cc1ccncc1)C1C(=O)N(Cc2ccncc2)C(=O)C31</smiles>

\section{Experimental}

2.1. Crystal data

$\mathrm{C}_{24} \mathrm{H}_{22} \mathrm{~N}_{4} \mathrm{O}_{4}{ }^{2+} \cdot 2 \mathrm{NO}_{3}{ }^{-}$

$M_{r}=554.48$

Monoclinic, $P 2_{1} / c$

$a=13.0706(6) \AA$

$b=14.3587(5) \AA$

$c=12.9893(5) \AA$

$\beta=104.861(4)^{\circ}$

$V=2356.25(16) \AA^{3}$

$Z=4$

Mo $K \alpha$ radiation

$\mu=0.12 \mathrm{~mm}^{-1}$

$T=153 \mathrm{~K}$

$0.30 \times 0.25 \times 0.20 \mathrm{~mm}$

\subsection{Data collection}

Bruker MWPC diffractometer Absorption correction: multi-scan (SADABS; Bruker, 2004) $T_{\min }=0.963, T_{\max }=0.976$

13323 measured reflections 4555 independent reflections 2623 reflections with $I>2 \sigma(I)$ $R_{\text {int }}=0.031$

\subsection{Refinement}

$R\left[F^{2}>2 \sigma\left(F^{2}\right)\right]=0.043$

$w R\left(F^{2}\right)=0.146$

$S=1.00$

4555 reflections

361 parameters

8 restraints

$\mathrm{H}$-atom parameters constrained

$\Delta \rho_{\max }=0.23 \mathrm{e}^{-3}$

$\Delta \rho_{\min }=-0.28 \mathrm{e}^{-3}$

Table 1

Hydrogen-bond geometry $\left(\AA{ }^{\circ}\right)$.

\begin{tabular}{lllll}
\hline$D-\mathrm{H} \cdots A$ & $D-\mathrm{H}$ & $\mathrm{H} \cdots A$ & $D \cdots A$ & $D-\mathrm{H} \cdots A$ \\
\hline $\mathrm{N} 1-\mathrm{H} 1 A \cdots \mathrm{O} 6^{\mathrm{i}}$ & 0.86 & 2.40 & $3.096(3)$ & 138 \\
$\mathrm{~N} 1-\mathrm{H} 1 A \cdots 7^{\mathrm{i}}$ & 0.86 & 1.90 & $2.728(3)$ & 161 \\
$\mathrm{~N} 4-\mathrm{H} 4 A \cdots 9^{\mathrm{ii}}$ & 0.86 & 1.93 & $2.771(3)$ & 164 \\
$\mathrm{C} 6-\mathrm{H} 6 B \cdots 3^{\mathrm{iii}}$ & 0.97 & 2.57 & $3.386(3)$ & 142 \\
$\mathrm{C} 8-\mathrm{H} 8 A \cdots \mathrm{O} 8$ & 0.98 & 2.30 & $3.155(3)$ & 145 \\
$\mathrm{C} 11-\mathrm{H} 11 A \cdots \mathrm{O}^{\mathrm{iv}}$ & 0.98 & 2.48 & $3.386(3)$ & 153 \\
$\mathrm{C} 13-\mathrm{H} 13 A \cdots 5^{\mathrm{v}}$ & 0.93 & 2.30 & $3.121(3)$ & 147 \\
$\mathrm{C} 14-\mathrm{H} 14 A \cdots \mathrm{O}^{\mathrm{iv}}$ & 0.98 & 2.54 & $3.403(3)$ & 147 \\
$\mathrm{C} 19-\mathrm{H} 19 A \cdots 6^{\mathrm{vi}}$ & 0.97 & 2.55 & $3.246(3)$ & 129 \\
$\mathrm{C} 21-\mathrm{H} 21 A \cdots 6^{\mathrm{vi}}$ & 0.93 & 2.56 & $3.362(3)$ & 145 \\
$\mathrm{C} 22-\mathrm{H} 22 A \cdots 2^{\text {vii }}$ & 0.93 & 2.43 & $3.257(3)$ & 149 \\
$\mathrm{C} 23-\mathrm{H} 23 A \cdots 7^{\mathrm{v}}$ & 0.93 & 2.56 & $3.345(3)$ & 143 \\
\hline
\end{tabular}

Symmetry codes: (i) $-x,-y,-z+1$; (ii) $x-1,-y+\frac{1}{2}, z-\frac{1}{2}$; (iii) $-x+1, y-\frac{1}{2},-z+\frac{1}{2}$; (iv) $x,-y+\frac{1}{2}, z-\frac{1}{2}$; (v) $x, y, z-1$; (vi) $-x,-y+1,-z+1$; (vii) $-x, y+\frac{1}{2},-z+\frac{1}{2}$.

Data collection: FRAMBO (Bruker, 2004); cell refinement: FRAMBO and SAINT (Bruker, 2004); data reduction: SAINT (Bruker, 2004); program(s) used to solve structure: SHELXS97 (Sheldrick, 2008); program(s) used to refine structure: SHELXL97 (Sheldrick, 2008); molecular graphics: SHELXTL (Sheldrick, 2008); software used to prepare material for publication: SHELXTL.

Supporting information for this paper is available from the IUCr electronic archives (Reference: SU5240)

\title{
References
}

Bruker (2004). FRAMBO, SAINT and SADABS. Bruker AXS Inc., Madison, Wisconsion, USA.

Li, G.-B., He, J.-R., Liu, J.-M. \& Su, C.-Y. (2012a). CrystEngComm, 14, $2152-$ 2158.

Li, G.-B., He, J.-R., Pan, M., Deng, H.-Y., Liu, J.-M. \& Su, C.-Y. (2012b). Dalton Trans. 41, 4626-4633. 


\section{data reports}

Li, G.-B., Liu, J.-M., Cai, Y.-P. \& Su, C.-Y. (2011). Cryst. Growth Des. 11, $2763-$ 2772.

Liu, Z.-M., Liu, Y., Zheng, S.-R., Yu, Z.-Q., Pan, M. \& Su, C.-Y. (2007). Inorg. Chem. 46, 5814-5816.
Sheldrick, G. M. (2008). Acta Cryst. A64, 112-122.

Yu, Z.-Q., Pan, M., Jiang, J.-J., Liu, Z.-M. \& Su, C.-Y. (2012). Cryst. Growth Des. 12, 2389-2396. 


\section{supporting information}

Acta Cryst. (2015). E71, o986-o987 [https://doi.org/10.1107/S2056989015022227]

Crystal structure of 4,4' -[(1,3,5,7-tetraoxo-1,3,3a,4,4a,5,7,7a,8,8a-decahydro-4,8-ethenopyrrolo[3,4-f] isoindole-2,6-diyl)bis(methylene)]bis(pyridin-1ium) dinitrate

\section{Zhimin Liu}

\section{S1. Comment}

In recent years, complexes with terminal pyridyl-substituted ligands have been used to construct various metal-organic frameworks (MOFs). MOFs have aroused considerable interests not only owing to their novel topological structures and for their potential applications (Li et al., 2011, 2012a,b; Yu et al., 2012). The title salt was synthesized from the reaction of

2,6-bis(pyridin-4-ylmethyl)-3a,4,4a,7a,8,8a-hexahydro-4,8-ethenopyrrolo[3,4-f]isoindole-1,3,5,7(2H,6H)-tetraone and nitric acid in chloroform.

In the title salt, Fig. 1, the cation has two terminal protonated pyridine N atoms, N1 and N4. The backbone of the cation has essentially a cis- $\mathrm{U}$ conformation. The two isoindole dione rings $(\mathrm{N} 2 / \mathrm{C} 7-\mathrm{C} 10$ and $\mathrm{N} 3 / \mathrm{C} 15-\mathrm{C} 18)$ are inclined to one another by $60.41(13)^{\circ}$, while the two outer pyridine rings ( $\mathrm{N} 1 / \mathrm{C} 1-\mathrm{C} 5$ and $\left.\mathrm{N} 4 / \mathrm{C} 20-\mathrm{C} 24\right)$ are inclined to one another by $2.77(12)^{\circ}$. The dihedral angles between the pyridine ring and the adjacent isoindole dione ring are $86.44(13)^{\circ}$ for rings $\mathrm{N} 1 / \mathrm{C} 1-\mathrm{C} 5$ and $\mathrm{N} 2 / \mathrm{C} 7-\mathrm{C} 10$, and $71.82(12)^{\circ}$ for rings $\mathrm{N} 4 / \mathrm{C} 20-\mathrm{C} 24$ and $\mathrm{N} 3 / \mathrm{C} 15-\mathrm{C} 18$. The bond angles $\mathrm{C} 3-$ $\mathrm{C} 6-\mathrm{N} 2$ and $\mathrm{C} 20-\mathrm{C} 19-\mathrm{N} 3$ are $114.1(2)$ and $113.52(19)^{\circ}$, respectively, larger than normal $\left(109^{\circ}\right)$

In the crystal, each anion is linked to a protonated pyridine ring by $\mathrm{N}-\mathrm{H}^{\cdots} \mathrm{O}$ hydrogen bonds (Table 1). These units are linked by a series of $\mathrm{C}-\mathrm{H} \cdots \mathrm{O}$ hydrogen bonds forming a three-dimensional structure (Table 1 and Fig. 2).

\section{S2. Synthesis and crystallization}

The compound 2,6-bis(pyridin-4-ylmethyl)-3a,4,4a,7a,8,8a-hexahydro-4,8-ethenopyrrolo[3,4-f]isoindole-1,3,5,7(2H,6H)-tetraone (L) was prepared according to a reported procedure (Liu et al., 2007). $\mathbf{L}$ (1 mmol, 0.43g) was added to $\mathrm{CHCl}_{3}(5 \mathrm{ml})$ under vigorous stirring. The clear solution was combined with nitric(V) acid $(0.1 \mathrm{M}, 1 \mathrm{ml})$ and stirred for $20 \mathrm{~min}$. The resulting solution was left to crystallize at ambient temperature. After two weeks, large blockshaped yellow single crystals of the title salt suitable for X-ray diffraction analysis were obtained [yield: $73 \%$; based on the 4-(aminomethyl)-pyridine].

\section{S3. Refinement details}

Crystal data, data collection and structure refinement details are summarized in Table 2. The $\mathrm{H}$ atoms were placed in calculated positions and refined in a riding-model approximation: $\mathrm{N}-\mathrm{H}=0.86 \AA$ and $\mathrm{C}-\mathrm{H}=0.93-0.98 \AA$ with $\mathrm{U}_{\text {iso }}(\mathrm{H})=$ $1.2 \mathrm{U}_{\mathrm{eq}}(\mathrm{N}, \mathrm{C})$. 

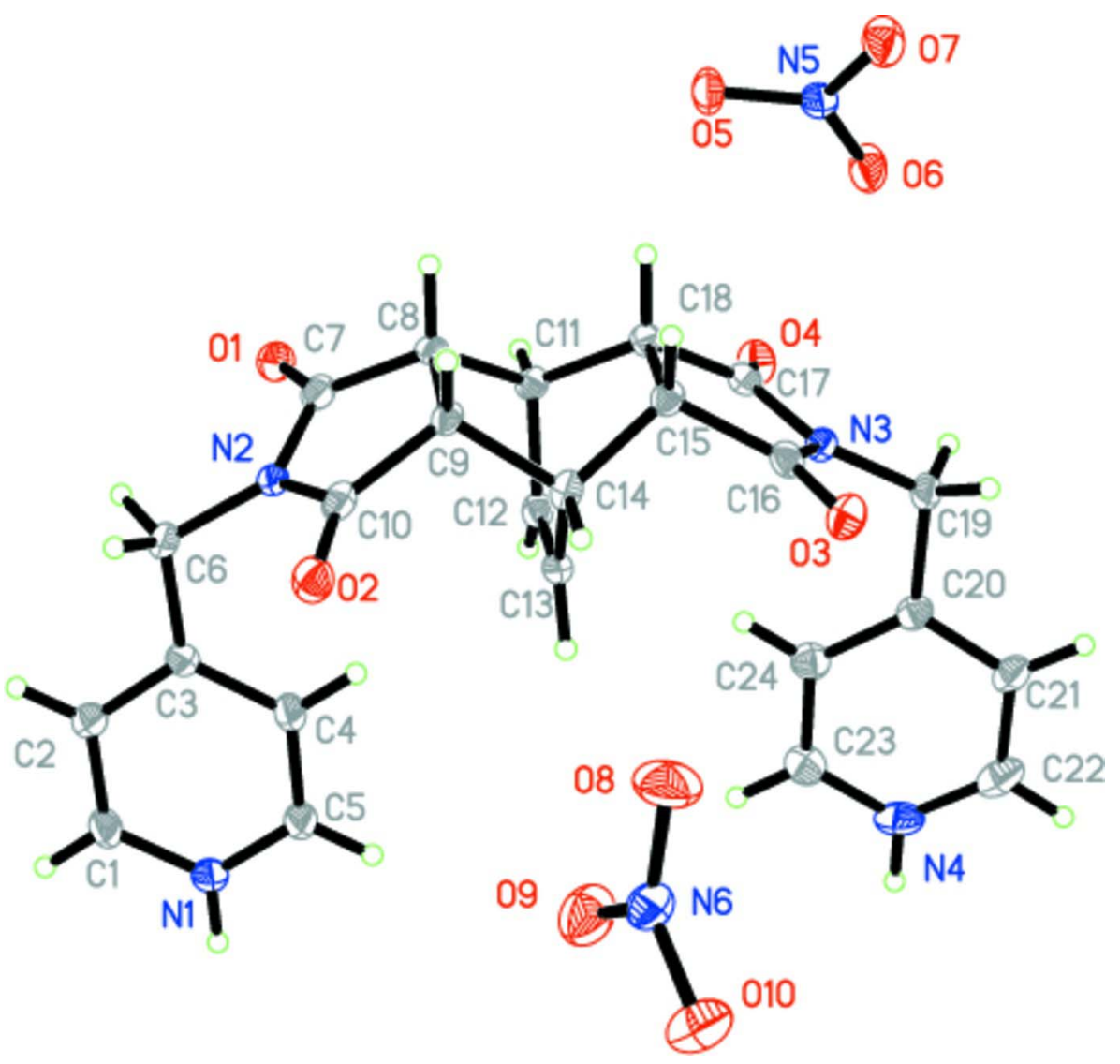

Figure 1

The molecular structure of the title salt, with atom labelling. Displacement ellipsoids are drawn at the $30 \%$ probability level.

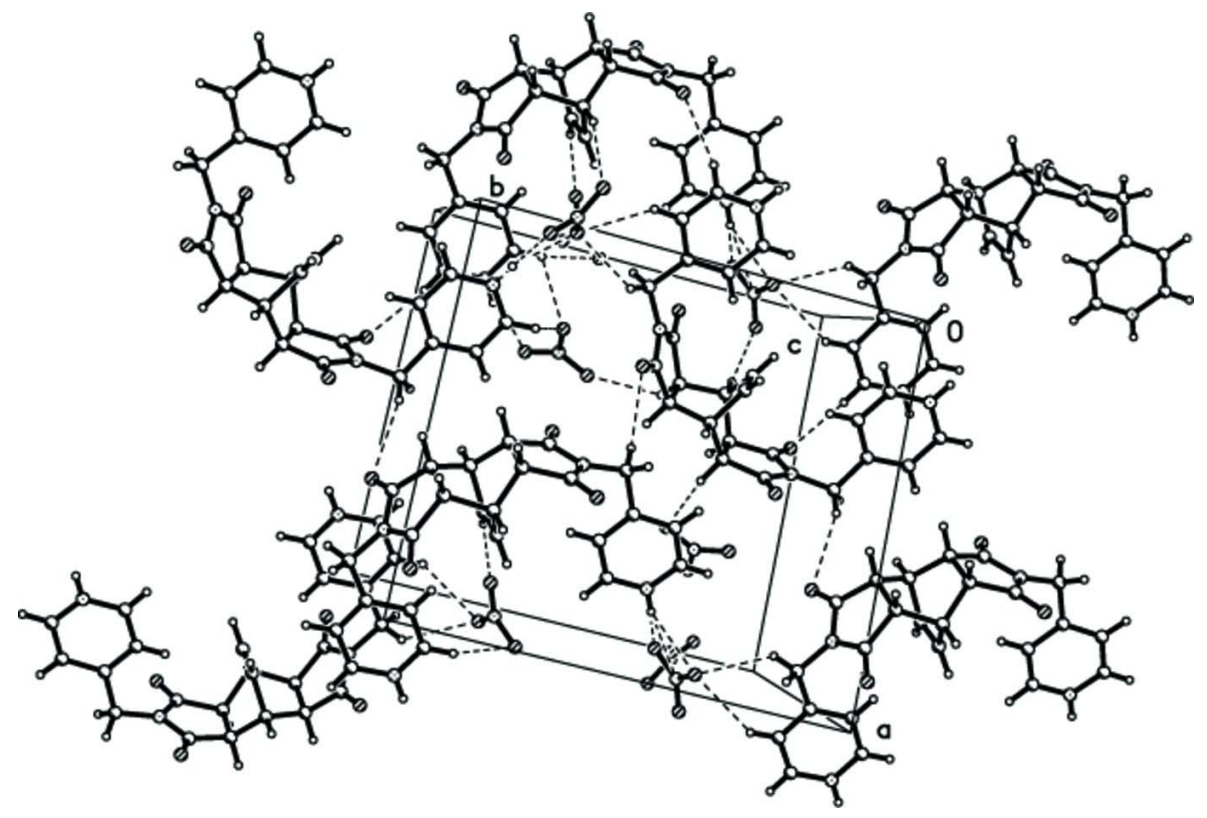


Figure 2

Crystal packing of the title salt, viewed along the $c$ axis. hydrogen bonds are shown as dashed lines (see Table 1).

4,4'-[(1,3,5,7-Tetraoxo-1,3,3a,4,4a,5,7,7a,8,8a-decahydro-4,8-ethenopyrrolo[3,4-f] isoindole-2,6-

diyl)bis(methylene)]bis(pyridin-1-ium) dinitrate

\section{Crystal data}

$\mathrm{C}_{24} \mathrm{H}_{22} \mathrm{~N}_{4} \mathrm{O}_{4}{ }^{2+} \cdot 2 \mathrm{NO}_{3}^{-}$

$M_{r}=554.48$

Monoclinic, $P 2_{1} / c$

Hall symbol: $-\mathrm{P} 2 \mathrm{ybc}$

$a=13.0706(6) \AA$

$b=14.3587(5) \AA$

$c=12.9893(5) \AA$

$\beta=104.861(4)^{\circ}$

$V=2356.25(16) \AA^{3}$

$Z=4$

\section{Data collection}

Bruker MWPC

diffractometer

Radiation source: fine-focus sealed tube

Graphite monochromator

Detector resolution: 16.08 pixels $\mathrm{mm}^{-1}$

phi and $\omega$ scans

Absorption correction: multi-scan

(SADABS; Bruker, 2004)

$T_{\min }=0.963, T_{\max }=0.976$

\section{Refinement}

Refinement on $F^{2}$

Least-squares matrix: full

$R\left[F^{2}>2 \sigma\left(F^{2}\right)\right]=0.043$

$w R\left(F^{2}\right)=0.146$

$S=1.00$

4555 reflections

361 parameters

8 restraints

Primary atom site location: structure-invariant direct methods
$F(000)=1152$

$D_{\mathrm{x}}=1.563 \mathrm{Mg} \mathrm{m}^{-3}$

Mo $K \alpha$ radiation, $\lambda=0.71073 \AA$

Cell parameters from 4348 reflections

$\theta=2.8-29.7^{\circ}$

$\mu=0.12 \mathrm{~mm}^{-1}$

$T=153 \mathrm{~K}$

Block, yellow

$0.30 \times 0.25 \times 0.20 \mathrm{~mm}$

13323 measured reflections

4555 independent reflections

2623 reflections with $I>2 \sigma(I)$

$R_{\text {int }}=0.031$

$\theta_{\text {max }}=26.0^{\circ}, \theta_{\min }=2.8^{\circ}$

$h=-16 \rightarrow 12$

$k=-17 \rightarrow 17$

$l=-15 \rightarrow 16$

\section{Special details}

Geometry. All e.s.d.'s (except the e.s.d. in the dihedral angle between two 1.s. planes) are estimated using the full covariance matrix. The cell e.s.d.'s are taken into account individually in the estimation of e.s.d.'s in distances, angles and torsion angles; correlations between e.s.d.'s in cell parameters are only used when they are defined by crystal symmetry. An approximate (isotropic) treatment of cell e.s.d.'s is used for estimating e.s.d.'s involving 1.s. planes.

Refinement. Refinement of $F^{2}$ against ALL reflections. The weighted $R$-factor $w R$ and goodness of fit $S$ are based on $F^{2}$, conventional $R$-factors $R$ are based on $F$, with $F$ set to zero for negative $F^{2}$. The threshold expression of $F^{2}>\sigma\left(F^{2}\right)$ is used only for calculating $R$-factors(gt) etc. and is not relevant to the choice of reflections for refinement. $R$-factors based on $F^{2}$ are statistically about twice as large as those based on $F$, and $R$ - factors based on ALL data will be even larger. 
Fractional atomic coordinates and isotropic or equivalent isotropic displacement parameters $\left(\hat{A}^{2}\right)$

\begin{tabular}{|c|c|c|c|c|}
\hline & $x$ & $y$ & $z$ & $U_{\text {iso }} * / U_{\text {eq }}$ \\
\hline $\mathrm{O} 1$ & $0.50387(14)$ & $0.20676(12)$ & $0.21910(13)$ & $0.0393(5)$ \\
\hline $\mathrm{O} 3$ & $0.28425(13)$ & $0.55976(12)$ & $0.17390(13)$ & $0.0359(4)$ \\
\hline $\mathrm{O} 4$ & $0.11752(14)$ & $0.46314(12)$ & $0.42145(14)$ & $0.0405(5)$ \\
\hline N6 & $0.70026(17)$ & $0.29400(14)$ & $0.54704(17)$ & $0.0345(5)$ \\
\hline $\mathrm{O} 2$ & $0.38605(14)$ & $0.12774(12)$ & $0.50720(13)$ & $0.0383(4)$ \\
\hline N3 & $0.18131(15)$ & $0.52033(12)$ & $0.28596(15)$ & $0.0274(5)$ \\
\hline N4 & $-0.16669(16)$ & $0.40594(14)$ & $0.05847(16)$ & $0.0330(5)$ \\
\hline $\mathrm{H} 4 \mathrm{~A}$ & -0.2196 & 0.3725 & 0.0256 & $0.040^{*}$ \\
\hline $\mathrm{O} 10$ & $0.75841(16)$ & $0.28012(13)$ & $0.63718(15)$ & $0.0479(5)$ \\
\hline $\mathrm{C} 7$ & $0.47472(18)$ & $0.21943(16)$ & 0.29881 (19) & $0.0288(5)$ \\
\hline $\mathrm{N} 2$ & $0.44842(15)$ & $0.14774(12)$ & $0.36012(15)$ & $0.0273(5)$ \\
\hline O9 & $0.68040(16)$ & $0.22794(12)$ & $0.48063(15)$ & $0.0507(5)$ \\
\hline $\mathrm{C} 20$ & $-0.00046(18)$ & $0.51242(16)$ & $0.16821(19)$ & $0.0281(5)$ \\
\hline $\mathrm{C} 14$ & $0.37646(18)$ & $0.37073(15)$ & $0.26654(17)$ & $0.0256(5)$ \\
\hline H14A & 0.4047 & 0.3876 & 0.2061 & $0.031 *$ \\
\hline C15 & $0.35286(18)$ & $0.45870(16)$ & $0.32628(17)$ & $0.0265(5)$ \\
\hline $\mathrm{H} 15 \mathrm{~A}$ & 0.4182 & 0.4932 & 0.3573 & $0.032 *$ \\
\hline O8 & $0.66218(15)$ & $0.37204(12)$ & $0.52061(15)$ & $0.0505(5)$ \\
\hline C3 & 0.36003 (19) & $0.00449(16)$ & $0.27263(18)$ & $0.0292(6)$ \\
\hline $\mathrm{C} 23$ & -0.0807 (2) & $0.36424(18)$ & 0.1159 (2) & $0.0365(6)$ \\
\hline $\mathrm{H} 23 \mathrm{~A}$ & -0.0773 & 0.2996 & 0.1185 & $0.044 *$ \\
\hline $\mathrm{C} 10$ & $0.41200(19)$ & $0.17942(17)$ & $0.44436(18)$ & $0.0282(5)$ \\
\hline C9 & $0.40865(18)$ & $0.28479(15)$ & $0.44104(18)$ & $0.0264(5)$ \\
\hline H9A & 0.4514 & 0.3109 & 0.5080 & $0.032 *$ \\
\hline $\mathrm{C} 4$ & $0.2756(2)$ & 0.05567 (19) & $0.21382(19)$ & $0.0345(6)$ \\
\hline H4B & 0.2780 & 0.1204 & 0.2161 & $0.041^{*}$ \\
\hline C6 & $0.46021(19)$ & $0.04991(15)$ & $0.3381(2)$ & $0.0307(6)$ \\
\hline H6A & 0.4835 & 0.0169 & 0.4052 & $0.037^{*}$ \\
\hline H6B & 0.5151 & 0.0435 & 0.3005 & $0.037^{*}$ \\
\hline C11 & $0.2933(2)$ & $0.32076(15)$ & 0.41775 (19) & $0.0297(6)$ \\
\hline H11A & 0.2586 & 0.3000 & 0.4723 & $0.036 *$ \\
\hline C18 & 0.29907 (18) & $0.42838(14)$ & $0.41363(17)$ & $0.0246(5)$ \\
\hline H18A & 0.3379 & 0.4531 & 0.4830 & $0.030 *$ \\
\hline $\mathrm{C} 2$ & $0.3535(2)$ & $-0.09187(16)$ & $0.2683(2)$ & $0.0348(6)$ \\
\hline $\mathrm{H} 2 \mathrm{~A}$ & 0.4078 & -0.1281 & 0.3092 & $0.042 *$ \\
\hline $\mathrm{C} 13$ & $0.27571(19)$ & $0.31613(15)$ & $0.23084(19)$ & $0.0295(6)$ \\
\hline $\mathrm{H} 13 \mathrm{~A}$ & 0.2449 & 0.3026 & 0.1596 & $0.035^{*}$ \\
\hline $\mathrm{C} 17$ & $0.1902(2)$ & $0.47097(15)$ & 0.37992 (19) & $0.0305(6)$ \\
\hline $\mathrm{C} 21$ & $-0.09112(18)$ & $0.55362(17)$ & 0.10493 (19) & $0.0326(6)$ \\
\hline $\mathrm{H} 21 \mathrm{~A}$ & -0.0960 & 0.6181 & 0.0997 & $0.039 *$ \\
\hline $\mathrm{C} 8$ & $0.45595(18)$ & $0.31102(16)$ & $0.34811(18)$ & $0.0262(5)$ \\
\hline H8A & 0.5228 & 0.3446 & 0.3744 & $0.031^{*}$ \\
\hline N1 & $0.18676(18)$ & $-0.08144(16)$ & $0.14709(18)$ & $0.0438(6)$ \\
\hline $\mathrm{H} 1 \mathrm{~A}$ & 0.1327 & -0.1085 & 0.1062 & $0.053 *$ \\
\hline C16 & 0.27339 (19) & $0.51993(16)$ & $0.2524(2)$ & $0.0295(5)$ \\
\hline
\end{tabular}




$\begin{array}{lllll}\text { C12 } & 0.23399(18) & 0.28860(15) & 0.30875(19) & 0.0305(6) \\ \text { H12A } & 0.1731 & 0.2524 & 0.2968 & 0.037^{*} \\ \text { C24 } & 0.0034(2) & 0.41598(17) & 0.1715(2) & 0.0393(7) \\ \text { H24A } & 0.0636 & 0.3862 & 0.2119 & 0.047^{*} \\ \text { C5 } & 0.1890(2) & 0.01172(19) & 0.1526(2) & 0.0392(6) \\ \text { H5A } & 0.1315 & 0.0463 & 0.1146 & 0.047^{*} \\ \text { C19 } & 0.08681(19) & 0.57240(16) & 0.2337(2) & 0.0316(6) \\ \text { H19A } & 0.1056 & 0.6191 & 0.1879 & 0.038^{*} \\ \text { H19B } & 0.0606 & 0.6046 & 0.2875 & 0.038^{*} \\ \text { C22 } & -0.1736(2) & 0.49923(17) & 0.0501(2) & 0.0365(6) \\ \text { H22A } & -0.2341 & 0.5268 & 0.0074 & 0.044^{*} \\ \text { C1 } & 0.2658(2) & -0.1332(2) & 0.2030(2) & 0.0454(7) \\ \text { H1B } & 0.2618 & -0.1977 & 0.1979 & 0.055^{*} \\ \text { N5 } & 0.01934(18) & 0.21064(14) & 0.97829(19) & 0.0381(5) \\ \text { O7 } & -0.03711(16) & 0.15681(13) & 1.01790(16) & 0.0491(5) \\ \text { O6 } & -0.01019(17) & 0.23019(13) & 0.88230(16) & 0.0542(6) \\ \text { O5 } & 0.10141(15) & 0.24289(15) & 1.03608(17) & 0.0569(6) \\ \end{array}$

Atomic displacement parameters $\left(\AA^{2}\right)$

\begin{tabular}{lllllll}
\hline & $U^{11}$ & $U^{22}$ & $U^{33}$ & $U^{12}$ & $U^{13}$ & $U^{23}$ \\
\hline O1 & $0.0449(11)$ & $0.0410(10)$ & $0.0353(10)$ & $0.0081(9)$ & $0.0161(9)$ & $0.0021(8)$ \\
O3 & $0.0375(10)$ & $0.0340(9)$ & $0.0353(10)$ & $0.0000(8)$ & $0.0075(8)$ & $0.0069(8)$ \\
O4 & $0.0428(11)$ & $0.0357(10)$ & $0.0486(11)$ & $0.0037(9)$ & $0.0220(9)$ & $-0.0010(9)$ \\
N6 & $0.0299(12)$ & $0.0288(11)$ & $0.0384(13)$ & $-0.0005(10)$ & $-0.0030(10)$ & $-0.0065(10)$ \\
O2 & $0.0471(11)$ & $0.0318(9)$ & $0.0365(10)$ & $0.0004(9)$ & $0.0115(9)$ & $0.0056(8)$ \\
N3 & $0.0234(11)$ & $0.0213(10)$ & $0.0335(11)$ & $-0.0013(8)$ & $0.0002(8)$ & $0.0004(9)$ \\
N4 & $0.0269(11)$ & $0.0307(11)$ & $0.0383(12)$ & $-0.0027(9)$ & $0.0028(10)$ & $-0.0045(10)$ \\
O10 & $0.0567(13)$ & $0.0399(11)$ & $0.0374(11)$ & $0.0075(10)$ & $-0.0058(10)$ & $-0.0023(9)$ \\
C7 & $0.0241(13)$ & $0.0294(13)$ & $0.0296(13)$ & $0.0005(11)$ & $0.0009(11)$ & $0.0015(11)$ \\
N2 & $0.0266(11)$ & $0.0220(10)$ & $0.0308(11)$ & $-0.0004(8)$ & $0.0025(9)$ & $-0.0005(8)$ \\
O9 & $0.0549(13)$ & $0.0311(10)$ & $0.0512(12)$ & $0.0098(9)$ & $-0.0139(10)$ & $-0.0130(9)$ \\
C20 & $0.0240(13)$ & $0.0282(13)$ & $0.0323(13)$ & $0.0001(10)$ & $0.0073(10)$ & $-0.0018(11)$ \\
C14 & $0.0291(13)$ & $0.0238(11)$ & $0.0235(12)$ & $-0.0006(10)$ & $0.0059(10)$ & $-0.0016(10)$ \\
C15 & $0.0224(12)$ & $0.0247(11)$ & $0.0279(12)$ & $-0.0040(9)$ & $-0.0014(9)$ & $0.0011(10)$ \\
O8 & $0.0499(12)$ & $0.0259(10)$ & $0.0589(13)$ & $0.0104(9)$ & $-0.0169(10)$ & $-0.0041(9)$ \\
C3 & $0.0328(14)$ & $0.0267(12)$ & $0.0293(13)$ & $-0.0033(11)$ & $0.0101(11)$ & $-0.0057(11)$ \\
C23 & $0.0309(15)$ & $0.0273(13)$ & $0.0459(16)$ & $0.0012(11)$ & $-0.0001(12)$ & $-0.0039(12)$ \\
C10 & $0.0266(13)$ & $0.0311(13)$ & $0.0245(13)$ & $0.0029(11)$ & $0.0022(11)$ & $0.0040(11)$ \\
C9 & $0.0302(14)$ & $0.0238(12)$ & $0.0242(12)$ & $-0.0007(10)$ & $0.0051(10)$ & $-0.0001(10)$ \\
C4 & $0.0341(15)$ & $0.0364(14)$ & $0.0313(14)$ & $-0.0014(12)$ & $0.0051(12)$ & $-0.0025(11)$ \\
C6 & $0.0338(15)$ & $0.0209(12)$ & $0.0356(14)$ & $0.0036(11)$ & $0.0055(11)$ & $-0.0013(11)$ \\
C11 & $0.0367(15)$ & $0.0221(11)$ & $0.0314(14)$ & $0.0030(11)$ & $0.0108(11)$ & $0.0025(10)$ \\
C18 & $0.0288(13)$ & $0.0192(11)$ & $0.0220(12)$ & $-0.0012(10)$ & $-0.0005(10)$ & $-0.0017(9)$ \\
C2 & $0.0405(16)$ & $0.0255(13)$ & $0.0420(15)$ & $-0.0034(12)$ & $0.0171(13)$ & $-0.0030(12)$ \\
C13 & $0.0311(14)$ & $0.0234(12)$ & $0.0295(13)$ & $0.0035(10)$ & $-0.0004(11)$ & $-0.0051(10)$ \\
C17 & $0.0372(15)$ & $0.0212(12)$ & $0.0322(13)$ & $-0.0012(11)$ & $0.0074(11)$ & $-0.0024(10)$ \\
C21 & $0.0309(15)$ & $0.0276(13)$ & $0.0366(14)$ & $-0.0014(11)$ & $0.0038(12)$ & $-0.0008(11)$
\end{tabular}


supporting information

\begin{tabular}{lllllll}
\hline C8 & $0.0249(13)$ & $0.0258(12)$ & $0.0261(12)$ & $-0.0008(10)$ & $0.0034(10)$ & $0.0027(10)$ \\
N1 & $0.0359(13)$ & $0.0504(15)$ & $0.0446(14)$ & $-0.0157(11)$ & $0.0093(11)$ & $-0.0168(11)$ \\
C16 & $0.0328(14)$ & $0.0235(12)$ & $0.0317(14)$ & $-0.0019(11)$ & $0.0073(11)$ & $-0.0012(11)$ \\
C12 & $0.0221(13)$ & $0.0216(12)$ & $0.0451(15)$ & $0.0014(10)$ & $0.0037(11)$ & $-0.0016(11)$ \\
C24 & $0.0296(15)$ & $0.0277(14)$ & $0.0526(17)$ & $0.0059(11)$ & $-0.0038(12)$ & $-0.0004(12)$ \\
C5 & $0.0379(16)$ & $0.0446(16)$ & $0.0337(15)$ & $-0.0001(13)$ & $0.0068(12)$ & $-0.0047(12)$ \\
C19 & $0.0317(14)$ & $0.0198(12)$ & $0.0406(14)$ & $0.0018(10)$ & $0.0042(11)$ & $0.0007(10)$ \\
C22 & $0.0303(15)$ & $0.0339(14)$ & $0.0400(15)$ & $0.0069(12)$ & $-0.0005(12)$ & $0.0008(12)$ \\
C1 & $0.0553(19)$ & $0.0345(15)$ & $0.0539(18)$ & $-0.0135(14)$ & $0.0276(15)$ & $-0.0112(13)$ \\
N5 & $0.0361(13)$ & $0.0255(11)$ & $0.0519(15)$ & $-0.0037(10)$ & $0.0099(11)$ & $-0.0078(11)$ \\
O7 & $0.0555(13)$ & $0.0414(11)$ & $0.0542(12)$ & $-0.0158(10)$ & $0.0212(10)$ & $-0.0075(9)$ \\
O6 & $0.0622(14)$ & $0.0435(12)$ & $0.0481(13)$ & $-0.0046(10)$ & $-0.0016(11)$ & $0.0059(10)$ \\
O5 & $0.0337(12)$ & $0.0590(13)$ & $0.0703(14)$ & $-0.0121(10)$ & $-0.0004(10)$ & $-0.0196(11)$ \\
& & & & & & \\
\hline
\end{tabular}

Geometric parameters $\left(\AA,{ }^{\circ}\right)$

\begin{tabular}{|c|c|c|c|}
\hline $\mathrm{O} 1-\mathrm{C} 7$ & $1.205(3)$ & $\mathrm{C} 9-\mathrm{C} 8$ & $1.537(3)$ \\
\hline $\mathrm{O} 3-\mathrm{C} 16$ & $1.209(3)$ & $\mathrm{C} 9-\mathrm{C} 11$ & $1.549(3)$ \\
\hline $\mathrm{O} 4-\mathrm{C} 17$ & $1.212(3)$ & C9-H9A & 0.9800 \\
\hline $\mathrm{N} 6-\mathrm{O} 10$ & $1.238(3)$ & $\mathrm{C} 4-\mathrm{C} 5$ & $1.360(3)$ \\
\hline $\mathrm{N} 6-\mathrm{O} 8$ & $1.238(3)$ & $\mathrm{C} 4-\mathrm{H} 4 \mathrm{~B}$ & 0.9300 \\
\hline N6-O9 & $1.263(2)$ & C6-H6A & 0.9700 \\
\hline $\mathrm{O} 2-\mathrm{C} 10$ & $1.214(3)$ & C6-H6B & 0.9700 \\
\hline $\mathrm{N} 3-\mathrm{C} 16$ & $1.381(3)$ & $\mathrm{C} 11-\mathrm{C} 12$ & $1.502(3)$ \\
\hline $\mathrm{N} 3-\mathrm{C} 17$ & $1.390(3)$ & $\mathrm{C} 11-\mathrm{C} 18$ & 1.549 (3) \\
\hline $\mathrm{N} 3-\mathrm{C} 19$ & $1.454(3)$ & $\mathrm{C} 11-\mathrm{H} 11 \mathrm{~A}$ & 0.9800 \\
\hline $\mathrm{N} 4-\mathrm{C} 23$ & $1.322(3)$ & $\mathrm{C} 18-\mathrm{C} 17$ & $1.507(3)$ \\
\hline $\mathrm{N} 4-\mathrm{C} 22$ & $1.345(3)$ & C18-H18A & 0.9800 \\
\hline $\mathrm{N} 4-\mathrm{H} 4 \mathrm{~A}$ & 0.8600 & $\mathrm{C} 2-\mathrm{C} 1$ & $1.373(4)$ \\
\hline $\mathrm{C} 7-\mathrm{N} 2$ & $1.397(3)$ & $\mathrm{C} 2-\mathrm{H} 2 \mathrm{~A}$ & 0.9300 \\
\hline $\mathrm{C} 7-\mathrm{C} 8$ & $1.510(3)$ & $\mathrm{C} 13-\mathrm{C} 12$ & $1.327(3)$ \\
\hline $\mathrm{N} 2-\mathrm{C} 10$ & $1.378(3)$ & $\mathrm{C} 13-\mathrm{H} 13 \mathrm{~A}$ & 0.9300 \\
\hline $\mathrm{N} 2-\mathrm{C} 6$ & $1.450(3)$ & $\mathrm{C} 21-\mathrm{C} 22$ & $1.373(3)$ \\
\hline $\mathrm{C} 20-\mathrm{C} 24$ & $1.386(3)$ & $\mathrm{C} 21-\mathrm{H} 21 \mathrm{~A}$ & 0.9300 \\
\hline $\mathrm{C} 20-\mathrm{C} 21$ & $1.389(3)$ & C $8-\mathrm{H} 8 \mathrm{~A}$ & 0.9800 \\
\hline $\mathrm{C} 20-\mathrm{C} 19$ & $1.506(3)$ & $\mathrm{N} 1-\mathrm{C} 1$ & $1.328(4)$ \\
\hline $\mathrm{C} 14-\mathrm{C} 13$ & $1.500(3)$ & $\mathrm{N} 1-\mathrm{C} 5$ & 1.339 (3) \\
\hline $\mathrm{C} 14-\mathrm{C} 8$ & $1.540(3)$ & N1-H1A & 0.8600 \\
\hline $\mathrm{C} 14-\mathrm{C} 15$ & $1.554(3)$ & $\mathrm{C} 12-\mathrm{H} 12 \mathrm{~A}$ & 0.9300 \\
\hline $\mathrm{C} 14-\mathrm{H} 14 \mathrm{~A}$ & 0.9800 & $\mathrm{C} 24-\mathrm{H} 24 \mathrm{~A}$ & 0.9300 \\
\hline $\mathrm{C} 15-\mathrm{C} 16$ & $1.504(3)$ & C5-H5A & 0.9300 \\
\hline $\mathrm{C} 15-\mathrm{C} 18$ & $1.543(3)$ & C19-H19A & 0.9700 \\
\hline $\mathrm{C} 15-\mathrm{H} 15 \mathrm{~A}$ & 0.9800 & C19-H19B & 0.9700 \\
\hline $\mathrm{C} 3-\mathrm{C} 4$ & $1.382(3)$ & $\mathrm{C} 22-\mathrm{H} 22 \mathrm{~A}$ & 0.9300 \\
\hline $\mathrm{C} 3-\mathrm{C} 2$ & $1.387(3)$ & $\mathrm{C} 1-\mathrm{H} 1 \mathrm{~B}$ & 0.9300 \\
\hline $\mathrm{C} 3-\mathrm{C} 6$ & $1.514(3)$ & N5-O5 & $1.231(3)$ \\
\hline $\mathrm{C} 23-\mathrm{C} 24$ & $1.369(3)$ & $\mathrm{N} 5-\mathrm{O} 6$ & $1.239(3)$ \\
\hline $\mathrm{C} 23-\mathrm{H} 23 \mathrm{~A}$ & 0.9300 & $\mathrm{~N} 5-\mathrm{O} 7$ & $1.265(3)$ \\
\hline
\end{tabular}


C10-C9

$\mathrm{O} 10-\mathrm{N} 6-\mathrm{O} 8$

O10- $\mathrm{N} 6-\mathrm{O} 9$

O8- 6 6- 09

$\mathrm{C} 16-\mathrm{N} 3-\mathrm{C} 17$

C16-N3-C19

C17-N3-C19

$\mathrm{C} 23-\mathrm{N} 4-\mathrm{C} 22$

$\mathrm{C} 23-\mathrm{N} 4-\mathrm{H} 4 \mathrm{~A}$

$\mathrm{C} 22-\mathrm{N} 4-\mathrm{H} 4 \mathrm{~A}$

$\mathrm{O} 1-\mathrm{C} 7-\mathrm{N} 2$

$\mathrm{O} 1-\mathrm{C} 7-\mathrm{C} 8$

$\mathrm{N} 2-\mathrm{C} 7-\mathrm{C} 8$

$\mathrm{C} 10-\mathrm{N} 2-\mathrm{C} 7$

$\mathrm{C} 10-\mathrm{N} 2-\mathrm{C} 6$

C7-N2-C6

$\mathrm{C} 24-\mathrm{C} 20-\mathrm{C} 21$

$\mathrm{C} 24-\mathrm{C} 20-\mathrm{C} 19$

$\mathrm{C} 21-\mathrm{C} 20-\mathrm{C} 19$

$\mathrm{C} 13-\mathrm{C} 14-\mathrm{C} 8$

$\mathrm{C} 13-\mathrm{C} 14-\mathrm{C} 15$

$\mathrm{C} 8-\mathrm{C} 14-\mathrm{C} 15$

C13-C14-H14A

C8-C14-H14A

$\mathrm{C} 15-\mathrm{C} 14-\mathrm{H} 14 \mathrm{~A}$

$\mathrm{C} 16-\mathrm{C} 15-\mathrm{C} 18$

$\mathrm{C} 16-\mathrm{C} 15-\mathrm{C} 14$

$\mathrm{C} 18-\mathrm{C} 15-\mathrm{C} 14$

C16-C15-H15A

C18-C15-H15A

C14-C15-H15A

$\mathrm{C} 4-\mathrm{C} 3-\mathrm{C} 2$

$\mathrm{C} 4-\mathrm{C} 3-\mathrm{C} 6$

$\mathrm{C} 2-\mathrm{C} 3-\mathrm{C} 6$

$\mathrm{N} 4-\mathrm{C} 23-\mathrm{C} 24$

$\mathrm{N} 4-\mathrm{C} 23-\mathrm{H} 23 \mathrm{~A}$

$\mathrm{C} 24-\mathrm{C} 23-\mathrm{H} 23 \mathrm{~A}$

$\mathrm{O} 2-\mathrm{C} 10-\mathrm{N} 2$

$\mathrm{O} 2-\mathrm{C} 10-\mathrm{C} 9$

N2- $\mathrm{C} 10-\mathrm{C} 9$

$\mathrm{C} 10-\mathrm{C} 9-\mathrm{C} 8$

$\mathrm{C} 10-\mathrm{C} 9-\mathrm{C} 11$

$\mathrm{C} 8-\mathrm{C} 9-\mathrm{C} 11$

$\mathrm{C} 10-\mathrm{C} 9-\mathrm{H} 9 \mathrm{~A}$

C8- C9-H9A

C11-C9-H9A

$\mathrm{C} 5-\mathrm{C} 4-\mathrm{C} 3$
$1.514(3)$

120.9 (2)

$119.6(2)$

$119.5(2)$

113.1 (2)

$124.03(19)$

122.77 (19)

121.7 (2)

119.1

119.1

$123.8(2)$

$128.1(2)$

$108.02(18)$

$113.26(19)$

$123.56(19)$

123.17 (19)

$117.6(2)$

122.5 (2)

$119.9(2)$

$107.79(18)$

$107.95(18)$

107.11 (17)

111.3

111.3

111.3

$105.29(18)$

$110.47(18)$

108.91 (17)

110.7

110.7

110.7

118.4 (2)

$122.3(2)$

$119.2(2)$

$120.2(2)$

119.9

119.9

$123.0(2)$

$128.2(2)$

$108.71(19)$

$104.63(18)$

111.01 (19)

$109.41(18)$

110.5

110.5

110.5

$120.2(2)$
C12-C11-H11A

C9-C11-H11A

C18-C11-H11A

C17-C18-C15

C17-C18-C11

C15-C18-C11

C17-C18-H18A

C15-C18-H18A

C11-C18-H18A

$\mathrm{C} 1-\mathrm{C} 2-\mathrm{C} 3$

$\mathrm{C} 1-\mathrm{C} 2-\mathrm{H} 2 \mathrm{~A}$

$\mathrm{C} 3-\mathrm{C} 2-\mathrm{H} 2 \mathrm{~A}$

$\mathrm{C} 12-\mathrm{C} 13-\mathrm{C} 14$

C12-C13-H13A

C14-C13-H13A

$\mathrm{O} 4-\mathrm{C} 17-\mathrm{N} 3$

$\mathrm{O} 4-\mathrm{C} 17-\mathrm{C} 18$

N3-C17-C18

$\mathrm{C} 22-\mathrm{C} 21-\mathrm{C} 20$

$\mathrm{C} 22-\mathrm{C} 21-\mathrm{H} 21 \mathrm{~A}$

$\mathrm{C} 20-\mathrm{C} 21-\mathrm{H} 21 \mathrm{~A}$

$\mathrm{C} 7-\mathrm{C} 8-\mathrm{C} 9$

C7-C $8-\mathrm{C} 14$

C9- 8 - $-\mathrm{C} 14$

$\mathrm{C} 7-\mathrm{C} 8-\mathrm{H} 8 \mathrm{~A}$

C9- 8 8- $\mathrm{H} 8 \mathrm{~A}$

$\mathrm{C} 14-\mathrm{C} 8-\mathrm{H} 8 \mathrm{~A}$

$\mathrm{C} 1-\mathrm{N} 1-\mathrm{C} 5$

$\mathrm{C} 1-\mathrm{N} 1-\mathrm{H} 1 \mathrm{~A}$

$\mathrm{C} 5-\mathrm{N} 1-\mathrm{H} 1 \mathrm{~A}$

$\mathrm{O} 3-\mathrm{C} 16-\mathrm{N} 3$

$\mathrm{O} 3-\mathrm{C} 16-\mathrm{C} 15$

N3-C16-C15

$\mathrm{C} 13-\mathrm{C} 12-\mathrm{C} 11$

$\mathrm{C} 13-\mathrm{C} 12-\mathrm{H} 12 \mathrm{~A}$

$\mathrm{C} 11-\mathrm{C} 12-\mathrm{H} 12 \mathrm{~A}$

$\mathrm{C} 23-\mathrm{C} 24-\mathrm{C} 20$

$\mathrm{C} 23-\mathrm{C} 24-\mathrm{H} 24 \mathrm{~A}$

$\mathrm{C} 20-\mathrm{C} 24-\mathrm{H} 24 \mathrm{~A}$

$\mathrm{N} 1-\mathrm{C} 5-\mathrm{C} 4$

$\mathrm{N} 1-\mathrm{C} 5-\mathrm{H} 5 \mathrm{~A}$

$\mathrm{C} 4-\mathrm{C} 5-\mathrm{H} 5 \mathrm{~A}$

N3-C19-C20

N3-C19-H19A

C20-C19-H19A

N3-C19-H19B
111.4

111.4

111.4

104.26 (18)

111.34 (19)

110.03 (18)

110.4

110.4

110.4

119.3 (3)

120.4

120.4

114.8 (2)

122.6

122.6

122.7 (2)

128.5 (2)

108.77 (19)

120.1 (2)

119.9

119.9

$105.16(18)$

110.32 (19)

109.90 (18)

110.4

110.4

110.4

121.7 (2)

119.1

119.1

124.4 (2)

127.1 (2)

108.45 (19)

114.4 (2)

122.8

122.8

120.5 (2)

119.7

119.7

119.9 (3)

120.1

120.1

113.52 (19)

108.9

108.9

108.9 


$\begin{array}{llll}\mathrm{C} 5-\mathrm{C} 4-\mathrm{H} 4 \mathrm{~B} & 119.9 & \mathrm{C} 20-\mathrm{C} 19-\mathrm{H} 19 \mathrm{~B} & 108.9 \\ \mathrm{C} 3-\mathrm{C} 4-\mathrm{H} 4 \mathrm{~B} & 119.9 & \mathrm{H} 19 \mathrm{~A}-\mathrm{C} 19-\mathrm{H} 19 \mathrm{~B} & 107.7 \\ \mathrm{~N} 2-\mathrm{C} 6-\mathrm{C} 3 & 114.1(2) & \mathrm{N} 4-\mathrm{C} 22-\mathrm{C} 21 & 119.8(2) \\ \mathrm{N} 2-\mathrm{C} 6-\mathrm{H} 6 \mathrm{~A} & 108.7 & \mathrm{~N} 4-\mathrm{C} 22-\mathrm{H} 22 \mathrm{~A} & 120.1 \\ \mathrm{C} 3-\mathrm{C} 6-\mathrm{H} 6 \mathrm{~A} & 108.7 & \mathrm{C} 21-\mathrm{C} 22-\mathrm{H} 22 \mathrm{~A} & 120.1 \\ \mathrm{~N} 2-\mathrm{C} 6-\mathrm{H} 6 \mathrm{~B} & 108.7 & \mathrm{~N} 1-\mathrm{C} 1-\mathrm{C} 2 & 120.4(3) \\ \mathrm{C} 3-\mathrm{C} 6-\mathrm{H} 6 \mathrm{~B} & 108.7 & \mathrm{~N} 1-\mathrm{C} 1-\mathrm{H} 1 \mathrm{~B} & 119.8 \\ \mathrm{H} 6 \mathrm{~A}-\mathrm{C} 6-\mathrm{H} 6 \mathrm{~B} & 107.6 & \mathrm{C} 2-\mathrm{C} 1-\mathrm{H} 1 \mathrm{~B} & 119.8 \\ \mathrm{C} 12-\mathrm{C} 11-\mathrm{C} 9 & 108.83(18) & \mathrm{O} 5-\mathrm{N} 5-\mathrm{O} 6 & 121.7(2) \\ \mathrm{C} 12-\mathrm{C} 11-\mathrm{C} 18 & 106.99(19) & \mathrm{O}-\mathrm{N} 5-\mathrm{O} 7 & 119.3(2) \\ \mathrm{C} 9-\mathrm{C} 11-\mathrm{C} 18 & 106.61(19) & \mathrm{O} 6-\mathrm{N} 5-\mathrm{O} 7 & 118.9(2)\end{array}$

Hydrogen-bond geometry $\left(\AA,{ }^{\circ}\right)$

\begin{tabular}{lllll}
\hline$D-\mathrm{H} \cdots A$ & $D-\mathrm{H}$ & $\mathrm{H} \cdots A$ & $D \cdots A$ & $D-\mathrm{H} \cdots A$ \\
\hline $\mathrm{N} 1-\mathrm{H} 1 A \cdots \mathrm{O} 6^{\mathrm{i}}$ & 0.86 & 2.40 & $3.096(3)$ & 138 \\
$\mathrm{~N} 1-\mathrm{H} 1 A \cdots \mathrm{O} 7^{\mathrm{i}}$ & 0.86 & 1.90 & $2.728(3)$ & 161 \\
$\mathrm{~N} 4-\mathrm{H} 4 A \cdots 9^{\mathrm{ii}}$ & 0.86 & 1.93 & $2.771(3)$ & 164 \\
$\mathrm{C} 6-\mathrm{H} 6 B \cdots \mathrm{O} 3^{\text {iii }}$ & 0.97 & 2.57 & $3.386(3)$ & 142 \\
$\mathrm{C} 8-\mathrm{H} 8 A \cdots \mathrm{O} 8$ & 0.98 & 2.30 & $3.155(3)$ & 145 \\
$\mathrm{C} 11-\mathrm{H} 11 A \cdots \mathrm{O} 5^{\text {iv }}$ & 0.98 & 2.48 & $3.386(3)$ & 153 \\
$\mathrm{C} 13-\mathrm{H} 13 A \cdots 5^{\mathrm{v}}$ & 0.93 & 2.30 & $3.121(3)$ & 147 \\
$\mathrm{C} 14-\mathrm{H} 14 A \cdots \mathrm{O} 2^{\text {iv }}$ & 0.98 & 2.54 & $3.403(3)$ & 147 \\
$\mathrm{C} 19-\mathrm{H} 19 A \cdots 6^{\text {vi }}$ & 0.97 & 2.55 & $3.246(3)$ & 129 \\
$\mathrm{C} 21-\mathrm{H} 21 A \cdots \mathrm{O} 6^{\text {vi }}$ & 0.93 & 2.56 & $3.362(3)$ & 145 \\
$\mathrm{C} 22-\mathrm{H} 22 A \cdots \mathrm{O} 2^{\text {vii }}$ & 0.93 & 2.43 & $3.257(3)$ & 149 \\
$\mathrm{C} 23-\mathrm{H} 23 A \cdots \mathrm{O} 7^{\mathrm{v}}$ & 0.93 & 2.56 & $3.345(3)$ & 143 \\
\hline
\end{tabular}

Symmetry codes: (i) $-x,-y,-z+1$; (ii) $x-1,-y+1 / 2, z-1 / 2$; (iii) $-x+1, y-1 / 2,-z+1 / 2$; (iv) $x,-y+1 / 2, z-1 / 2$; (v) $x, y, z-1$; (vi) $-x,-y+1,-z+1$; (vii) $-x$, $y+1 / 2,-z+1 / 2$. 